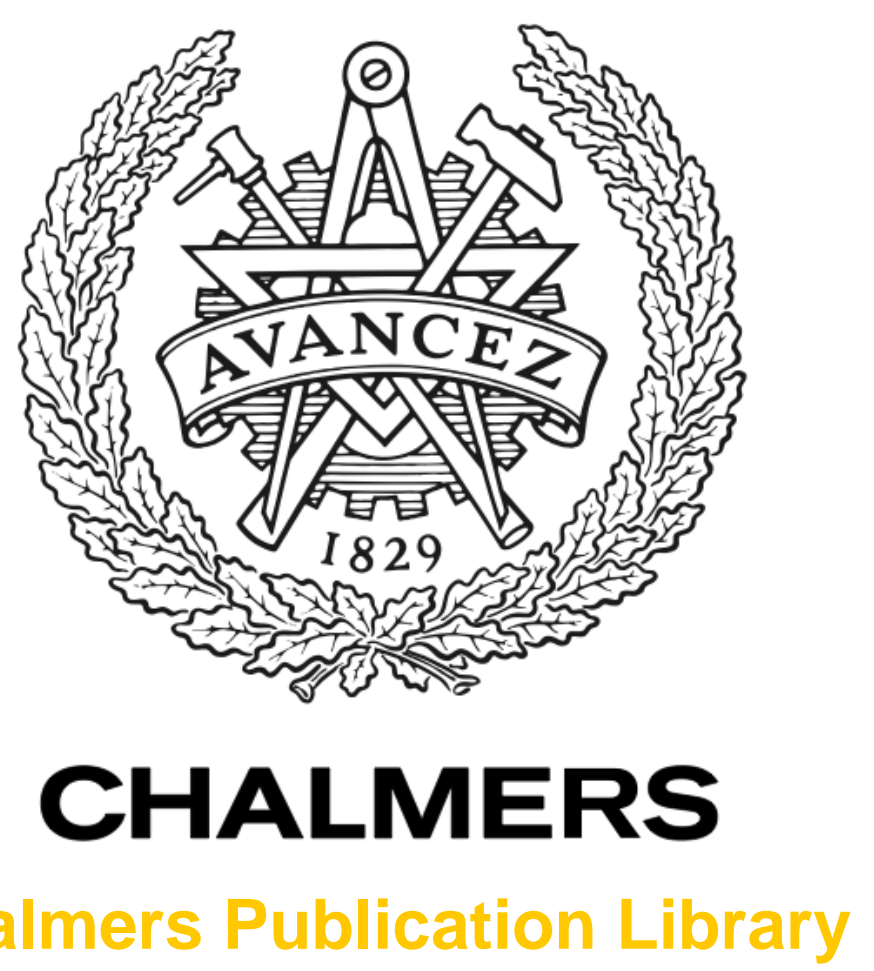

\title{
CMA-Based CD and DGD Estimation in Presence of Experimental Higher Order PMD
}

This document has been downloaded from Chalmers Publication Library (CPL). It is the author's version of a work that was accepted for publication in:

\section{European Conference on Optical Communications (ECOC 2014)}

Citation for the published paper:

Zakrisson, D. ; Karlsson, M. ; Johannisson, P. (2014) "CMA-Based CD and DGD Estimation in Presence of Experimental Higher Order PMD". European Conference on Optical

Communications (ECOC 2014) pp. P.3.16.

Downloaded from: http://publications.lib.chalmers.se/publication/207758

Notice: Changes introduced as a result of publishing processes such as copy-editing and formatting may not be reflected in this document. For a definitive version of this work, please refer to the published source. Please note that access to the published version might require a subscription. 


\title{
CMA-Based CD and DGD Estimation in Presence of Experimental Higher Order PMD
}

\author{
Daniel Zakrisson $^{(1)}$, Magnus Karlsson ${ }^{(1)}$, Pontus Johannisson (1) \\ (1) Photonics Lab, Department of Microtechnology and Nanoscience, Chalmers University of \\ Technology, SE-412 96 Gothenburg, Sweden, daniel.zakrisson@chalmers.se
}

\begin{abstract}
We evaluate 3 methods for CD estimation using CMA filter tap coefficients. The performance of these methods are evaluated with respect to their accuracy and range. We also experimentally evaluate the $C D$ estimation performance in presence of higher order PMD.
\end{abstract}

\section{Introduction}

The new generation of coherent optical communication systems are becoming wellestablished, which gives the advantage of using digital signal processing (DSP) for equalization and detection ${ }^{1}$. An adaptive equalizer, such as the constant modulus algorithm (CMA), is the subsystem in the digital coherent receiver that performs polarization demultiplexing and compensates for linear impairments, i.e. residual chromatic dispersion (CD), polarization mode dispersion (PMD), and polarization dependent loss $(P D L)^{1-2}$. These impairments that can be compensated for can therefore also be estimated from the finite impulse response (FIR) filter in the $\mathrm{CMA}^{1-4}$. The main advantage of estimating CD and PMD from the CMA FIR filter is that very little extra complexity is added to the DSP since we take advantage of a component already in the DSP. A CMA filter with hundreds of taps, long enough to compensate for several thousand kilometers of SMF (single mode fiber) dispersion, might be unrealistically complex. The CMA scheme is therefore preferably used for monitoring in compensated links.

In this paper we make two novel contributions. Firstly, in addition to the well known frequency-domain estimate ${ }^{1}$ we propose and experimentally evaluate two new estimates based on the time domain CMA coefficients. The three schemes are compared numerically and experimentally and shown to be advantageous in different regimes. Secondly, we perform for what we believe the first time, a recirculating loop experiment with a tuneable birefringent element in the loop, thus simultaneously estimating both CD and DGD in presence of randomly varying higher-order PMD.

\section{Theory}

A typical PM-QPSK coherent transmitter sends complex-valued signals in both polarizations. The channel can be expressed as $\boldsymbol{r}(f)=$ $\boldsymbol{H}(f) \boldsymbol{s}(f)+\boldsymbol{n}(f)$ where $\boldsymbol{r}(f)$ is the received signal spectrum, $\boldsymbol{H}(f)$ is the channel transfer matrix, $\boldsymbol{s}(f)$ is the transmitted signal spectrum and $\boldsymbol{n}(f)$ corresponds to additive white Gaussian noise. If the channel memory does not exceed the filter length, the CMA filter $\boldsymbol{W}\left(f_{n}\right)$ can be assumed to approximate the matched filter, i.e. $\boldsymbol{W}\left(f_{n}\right) \approx \boldsymbol{H}^{\dagger}\left(f_{n}\right) H_{p}^{*}\left(f_{n}\right)$, although strictly speaking the filter minimizes the CMA cost function. Here $H_{P}\left(f_{n}\right)$ is the signal spectral density which corresponds to the pulse shaping filter, the subscript $n$ represents the discrete coefficients of the FIR filter.

To find the transfer matrix $\boldsymbol{W}\left(f_{n}\right)$ we simply calculate the element-wise discrete Fourier transform of the finite impulse response (FIR) filter bank $\boldsymbol{W}\left(t_{n}\right)$. After canceling out the contributions of PMD by calculating the determinant of $\boldsymbol{W}\left(f_{n}\right)$ leaving only the common phase term accounting for the CD, taking the argument will give us the quadratic phase dependence of CD,

$$
\arg \left(\operatorname{det}\left(\mathbf{W}\left(f_{n}\right)\right)\right)=-\mathrm{n}^{2} \varphi_{\mathrm{FD}}
$$

where $n$ corresponds to the discrete frequency domain (FD) FIR filter tap number and $\varphi_{F D}$ is a constant related to the dispersion. Rewriting the right hand side of Eq. (1), using the normalized dispersion parameter $\delta=\beta_{2} z / T_{S}^{2}$ where $T_{s}$ is the sampling period,

$$
\varphi_{\mathrm{FD}}=-\delta \frac{(2 \pi)^{2}}{N^{2}}
$$

will be the dimension-less expression corresponding to the residual CD where $\mathrm{N}$ is the filter length. This is the conventional model suggested by ${ }^{1}$ which will be called the "FD phase model".

The residual CD will be compensated by the time domain (TD) FIR filter and its effect on the amplitude response will then correspond to the broadening of the initial pulse due to $C D$, this will be called the "TD amplitude model".

$$
\hat{\delta}_{11}=\arg \min _{\delta, \Delta \mathrm{n}}\left(\sum_{n=0}^{N}\left(\left|W_{11}\left(t_{n}\right)\right|-\mid d_{n}(\delta, \Delta n)\right)^{2}\right)
$$

In Eq. (3) $d_{n}(\delta, \Delta n)$ are samples of a CDbroadened Gaussian function where $\delta$ is the 
dispersion parameter and $\Delta n$ is a small shift that is needed since we don't know exactly where the center of the filter amplitude is. The minimization in Eq. (3) is done for all four components of $\boldsymbol{W}\left(t_{n}\right)$ and from the estimated $\hat{\delta}_{11}, \hat{\delta}_{12}, \hat{\delta}_{21}$ and $\hat{\delta}_{22}$ we can estimate the CD. We anticipate that this approach can provide reasonable estimates of the $C D$ even if the transmitted pulses are not strictly Gaussian.

In addition to the amplitude in the TD we can also use the phase for $C D$ estimation by taking the argument of the determinant of the TD FIR filter taps. Assuming sufficiently high dispersion so that the phase shift around the center taps are not too large, we get $\delta \approx 1 / \varphi_{T D}$, This will be the "TD phase model"

To estimate the DGD we start by normalizing with the square root of its determinant to remove the common phase term from the impulse response filter leaving the effects of DGD and PDL, $\boldsymbol{U}(f)=\boldsymbol{W}(f) / \sqrt{\operatorname{det}(\boldsymbol{W}(f))}$. The next step is to derive the matrix $\boldsymbol{U}(f)$ with respect to frequency and then take the determinant,

$$
\tau_{\mathrm{T}}=\tau_{\mathrm{DGD}} / T=2 \sqrt{\operatorname{det}\left(\frac{d \mathbf{U}(f)}{d f}\right)} / \mathrm{T}
$$

which will remove the effect of $\mathrm{PDL}^{1}$. We normalize with the symbol period $T$ to get the instantaneous DGD in units of the symbol period.

\section{Experimental evaluation of the CD estimation models}

In Fig. 1 we summarize the results from a large number of experimental measurements. The mean value and relative error in the estimated $C D$ is shown as a function of dispersion for the three methods evaluated over 100 independent measurements for each point. Simulations with

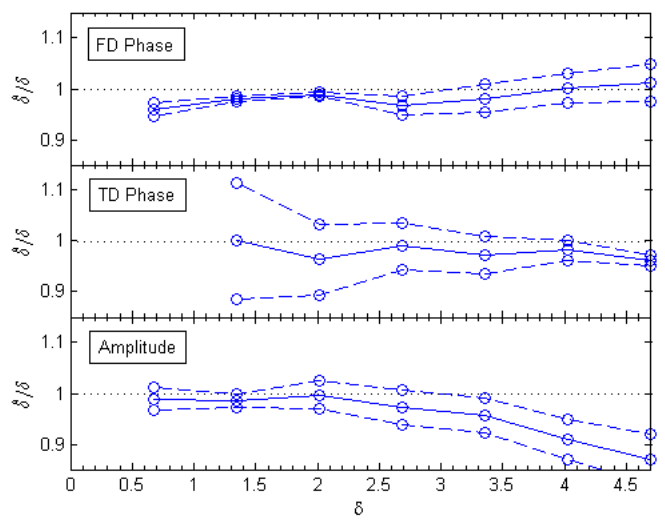

Fig. 1: Experimental relative mean value (solid line) and \pm one standard deviation (dashed lines) of CD estimation for all three models at a noise level corresponding to $10 \mathrm{~dB}$ OSNR. The vertical axis gives the relative deviation of the mean value of the estimated $C D$, and the corresponding standard deviation. similar conditions were made and gave comparable results, which are not shown here.

For the TD phase model case where $\delta \approx 1 / \varphi_{T D}$, the situation will be opposite to the FD case (Eq. (2)). At large accumulated CD the TD phase model will have a slow-changing phase response, and will outperform the FD model. At low CD the phase response will be too sharp to track and the FD phase model is preferred. The upper limit of the TD phase model will be determined by the limitations of the dynamic equalizer filter length and not the estimation method.

When estimating CD using the TD amplitude method one can always use all the taps. The limitations of the amplitude model are not as obvious as for the other two. There will be a point where the FIR tap amplitudes start to become non-Gaussian, which will have negative impact on the CD estimation. The two phase models are each preferable in different regimes whereas the amplitude model copes fairly well over the whole range. Also the amplitude model is robust to unwrapping errors in the phase estimation, which limits the performance of the phase-based methods.

\section{CD estimation in presence of higher order PMD}

In the experimental evaluation of the CD estimation performance in presence of higher order PMD we have used 28 Gbaud PM-QPSK data with a sampling rate of $100 \mathrm{GHz}$. To experimentally generate higher order differential group delay (DGD) we have used a circulating loop containing $160 \mathrm{~km}$ SMF, a tunable PMD emulator and a polarization scrambler that makes a random rotation of the polarization between each roundtrip in the loop. The emulator creates a tuneable first-order DGD, that was varied between 0 to 25 ps. The data was sent 13 laps in the loop giving a concatenation of 13 birefringent segments with random polarization rotation. For each setting on

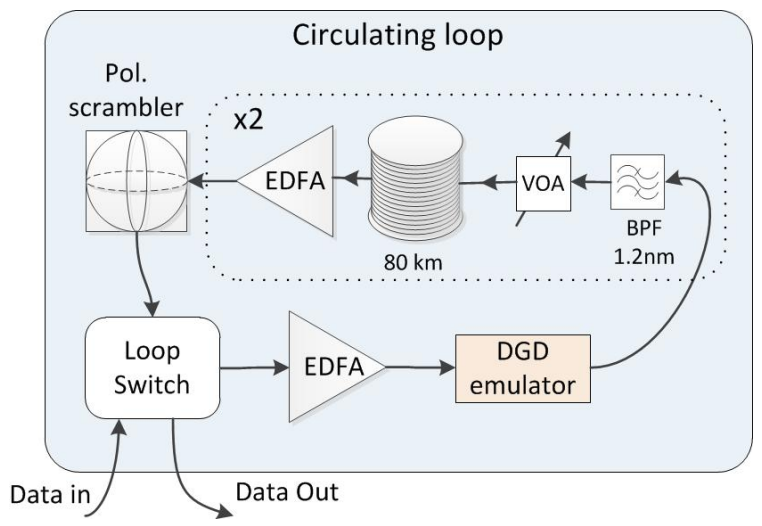

Fig. 2: Shows the circulating loop with the tunable first-order DGD emulator. 


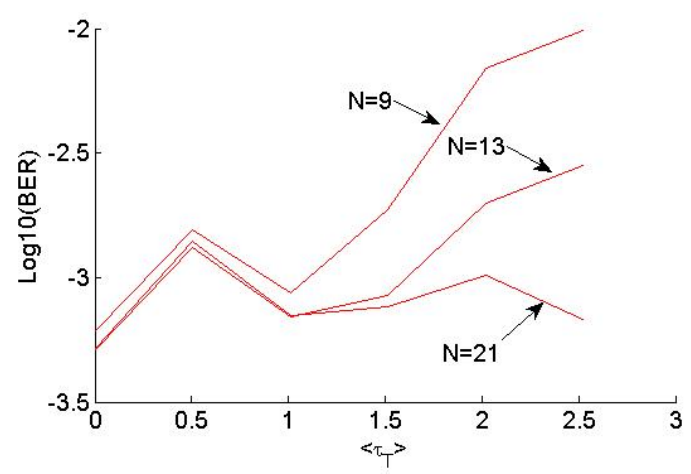

Fig. 3: BER vs avrage DGD for different CMA filter lengths $N$. The DGD is in units of symbol slots.

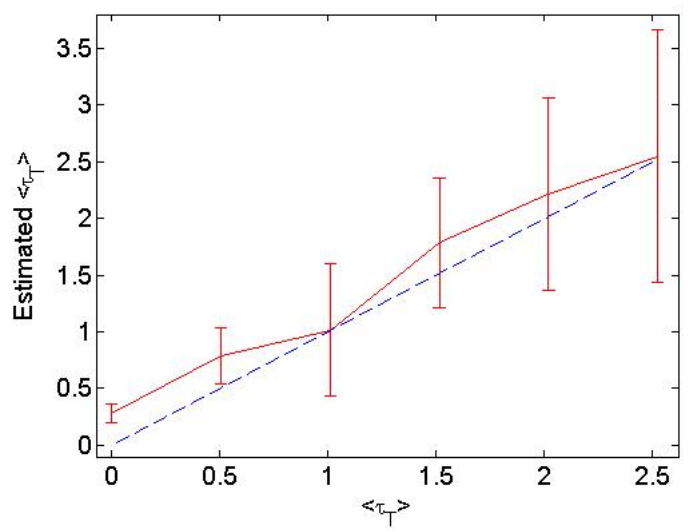

Fig. 4: DGD estimation of experimentally generated higher order DGD with bars that indicates the standard deviation. The dashed line is a reference line.

the PMD emulator 25 independent measurements were made. Although this statistical sample is small, it gives a hint on the standard deviation of the estimate. The experimental setup of the circulating loop can be seen in Fig. 2.

To stress test the CMA with respect to DGD compensation we reduced the filter length and monitored the BER, the results can be seen in Fig. 3. From the figure we can se that for a filter length of $\mathrm{N}=21$ CMA can compensate $\mathrm{DGD}$ corresponding to more than two symbol-slots without BER penalty, whereas for $\mathrm{N}=9$ we can see a BER penalty starting from an average DGD of one symbol-slot. Therefore we used $\mathrm{N}=21$ in the below estimates.

The average estimated DGD versus average DGD is shown in Fig. 4. The error bars indicates increasing standard deviation with increasing DGD, which is to be expected for higher order DGD. The offset from the reference line in Fig. 4 comes from of Eq. (4) which makes any estimation errors increase the result ${ }^{1}$.

From Fig. 5 we can see that for $C D$ estimation in presence of DGD, the amount of DGD have little or no effect on the performance of the $C D$ estimation which in agreement with results from ${ }^{2}$, and experiments (with different PMD statstics) in ${ }^{5,6}$.

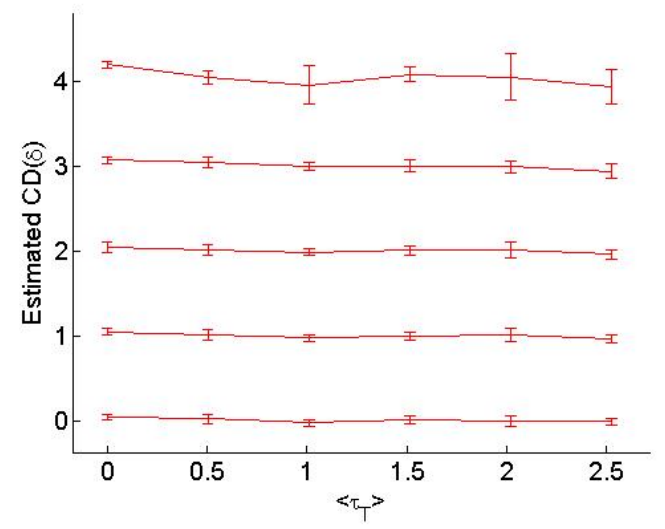

Fig. 5: Estimated $C D$ in prescence of higher order PMD using FD phase method, 21 CMA taps, bars shows standerd deviation. The amount of $C D$ corresponds to $\delta \approx 0,1,2,3,4$.

\section{Conclusions}

We have proposed 2 new TD methods for CD estimation from CMA FIR filters and evaluated these plus the conventional FD method from zero to high amount of CD to see at what regions they perform better than the others. The FD phase model is best for low amount of CD and the TD phase model is best for high amount of $C D$. The TD amplitude model works fairly well for both low and high amounts of CD.

True random and varying higher orders of PMD was emulated and estimeted experimentally for the first time, and the CD estimate was shown to be unaffected by the PMD, provided the number of tap coefficients are sufficient to provide reasonable BER.

\section{References}

[1] F. N. Hauske et al., "Optical Performance Monitoring in Digital Coherent Receivers" J. Lightwave Technol., Vol. 27, no. 16, p. 3623 (2009).

[2] F. N. Hauske et al., "Optical Performance Monitoring from FIR Filter Coefficients in Coherent Receivers," Proc. OFC, OThW2, San Diego (2008).

[3] G. Bosco et al., "Joint DGD, PDL and Chromatic Dispersion Estimation in Ultra-Long-Haul WDM Transmission Experiments with Coherent Receivers," Proc. ECOC, Th.10.A.2, Torino (2010).

[4] F. N. Hauske et al., "DGD Estimation from FIR Filter Taps in Presence of Higher Order PMD," Proc. ECOC, Tu.1.D.4, Brussels (2008)

[5] S. L. Woodward et al., "A Shared PMD and Chromatic Dispersion Monitor Based on a Coherent Receiver" Photon. Technol. Lett., Vol. 22, no. 10, p. 706 (2010).

[6] S. L. Woodward et al., "Characterization of Real-Time PMD and Chromatic Dispersion Monitoring in a HighPMD 46-Gb/s Transmission System" Photon. Technol. Lett., Vol. 20, no. 24, p. 2048 (2008) 